\title{
Evaluation of the effects of Ankaferd haemostat application on bone regeneration in rats with calvarial defects: histochemical, immunohistochemical and scintigraphic study
}

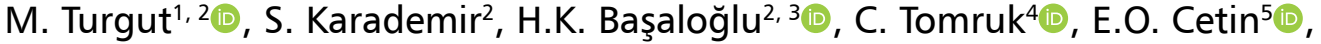

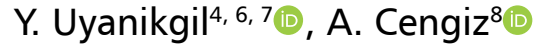 \\ ${ }^{1}$ Department of Neurosurgery, Aydın Adnan Menderes University, Faculty of Medicine, Aydın, Turkey \\ 2Department of Histology and Embryology, Aydın Adnan Menderes University, Health Sciences Institute, Aydın, Turkey \\ ${ }^{3}$ Department of Histology and Embryology, Aydın Adnan Menderes University, Faculty of Medicine, Aydın, Turkey \\ ${ }^{4}$ Department of Histology and Embryology, Ege University, Faculty of Medicine, Izmir, Turkey \\ ${ }^{5}$ Department of Pharmaceutical Technology, Department of Biopharmaceutics and Pharmacokinetics, \\ Ege University Faculty of Pharmacy, Izmir, Turkey \\ ${ }^{6}$ Department of Stem Cell, Ege University, Health Science Institue, Izmir, Turkey \\ ${ }^{7}$ Cord Blood, Cell and Tissue Research and Application Centre, Ege University, Izmir, Turkey \\ ${ }^{8}$ Department of Nuclear Medicine, Aydın Adnan Menderes University, Faculty of Medicine, Aydın, Turkey
}

[Received: 31 May 2021; Accepted: 9 July 2021; Early publication date: 3 August 2021]

Background: Bone wax, a haemostatic agent, is widely used in craniospinal surgical procedures for a long time, in spite of controversial results regarding its negative influence upon bone regeneration. In this experimental study, the effects of Ankaferd Blood Stopper (ABS), as an alternative haemostatic agent, were evaluated through histochemical, immunohistochemical and scintigraphic studies.

Materials and methods: The total of 30 adult female Wistar albino rats was randomly divided into three groups: intact control group $(n=10)$, bone wax group $(n=10)$, and ABS group $(n=10)$. Surgically, a $3.0 \mathrm{~mm}$ hole in diameter was drilled on the right side of calvarium of the rats using a Class Mini Grinder set in all three groups, as described previously. At the end of 8 weeks, bone healing and connective tissue alterations surrounding drilled calvarial defect areas of the rats were determined via haematoxylin and eosin and the Mallory's trichrome staining and anti-bone sialoprotein immunohistochemistry. Image Pro Express 4.5 programme was used for histomorphometric calculation of new bone and fibrotic tissue areas. All statistical analyses were made with SPSS 25.0 and analysis of variance (one-way ANOVA) followed by Bonferroni post hoc test was performed, $p<0.001$ was considered as significance level. Results: Histomorphometrically, it was found that he had the largest hole diameter and the least fibrotic scar area in the bone-wax group. In the bone wax group, it was observed that the material closed the hole and there was only a fibrotic scar tissue in the area between the bone tissue at the edge of the hole and bone wax, and a fibrotic tissue was formed in the bone wax area. During the histological procedure, this bone-wax material was poured and the sections were seen as a gap in this area. In the ABS haemostat group, the smallest hole diameter and the least fibrotic scar tissue were observed. Fibrotic scar tissue close to each other was found in the ABS haemostat and bone wax groups. Histological analysis of 
samples also showed a statistical significance for fibrotic connective tissue area between groups $(p<0.05)$. Scintigraphically, osteoblastic activity related to blood flow in the animal taken from the group with application of ABS haemostat was more pronounced compared to the other two groups.

Conclusions: In our study, it has been concluded that the ABS yields affirmative effects on the bone healing, while bone wax leads to negative impact on the bone regeneration. Scintigraphic, histochemical and immunohistochemical data support the affirmative impact of the ABS haemostat application upon the bone regeneration apart from the quick stop of haemorrhage. (Folia Morphol 2022; 81, 3: 739-748)

Key words: Ankaferd Blood Stopper haemostat, bone wax, calvarium, osteogenesis, rat

\section{INTRODUCTION}

Today, cranial defect is one of the most frequently encountered problems in craniofacial reconstructive surgery, but there is still controversy about repair of cranial defects. Bone wax, which is frequently used as a haemostatic agent in the control of bone bleeding in surgical operations, is the oldest known and cheapest absorbable substance prepared by Horsley in 1892 [see 1, 14]. It is a mixture of bee wax and isopropyl palmitate and is used in small pieces. Bone wax, which does not have any coagulation mechanism, stops the osseous bleeding in a physical way and it must be removed from the cavity properly after application because it delays bone regeneration [21, 31]. Therefore, it has been suggested that this material should not be used in places exposed to pressure and expected to heal quickly. On the other hand, Ankaferd Blood Stopper (ABS), consisting of Thymus vulgaris (thyme), Glycyrrhiza glabra (liquorice), Vitis vinifera (grape vine), Alpinia officinarum (blue ginger), and Urtica dioica (nettle) plant extracts, has been used as an alternative haemostatic agent in folk medicine for many years $[5,6,12]$. Recently, it has been widely used in some clinical trials for various tissue injuries as a topical agent for control of minor or major haemorrhages that occur spontaneously, such as gastrointestinal bleeding, or during or following some surgical interventions, including adenoidectomy and thyroidectomy $[5,7]$. Thus, it is well-known that it has a very rapid haemostatic effect in clinical practice, but few studies have assessed the influence of ABS haemostat on connective tissue and bone healing. In a previous experimental study by Bulut et al. (2014) [4], it has been reported that use of ABS as a haemostatic agent caused a negative effect on proliferation, number, viability and morphology of fibroblast cells. In similar, Gul et al. (2020) [13] found that fibrosis was significantly higher in the ABS group in mucosal tissue, suggesting its positive effect on wound healing in rats.

Recent studies have revealed that bone sialoprotein (BSP) and osteocalcin (OC) are expressed with the initiation of mineralisation with type I, type II and type $X$ collagens in the early period of bone formation $[18,29]$. In past, some researchers have suggested that bone formation is reduced by impaired mineralisation in mice with BSP deficiency [18]. Furthermore, it has also been suggested that osteonectin (ON) and alkaline phosphatase (AP) in cells have important roles in bone healing process. Due to their effects on bone cell differentiation and mineral formation, therefore, ABS haemostat can be used for stimulation of bone regeneration and its effects upon bone cells such as osteoblasts and osteoclasts will be determined, if noncollagenous bone related proteins BSP, OC, ON and osteopontin (OP) are evaluated immunohistochemically as bone formation markers.

On the other hand, three-phase bone scintigraphy is a non-invasive technique that shows the vascularity and osteoblastic activity of the bone. In their study using different bone grafts, Aygit et al. (1999) [2] evaluated vascularity and osseous changes with bone scintigraphy. In this study, they noted that bone scintigraphy could be used as a non-invasive method to evaluate implant vascularity because of histological changes and scintigraphic findings.

Therefore, the objective of this study was to analyse the changes that occur in the bone tissue of rats subjected to bone wax and ABS haemostat applications, which are used as haemostatic agents in various 

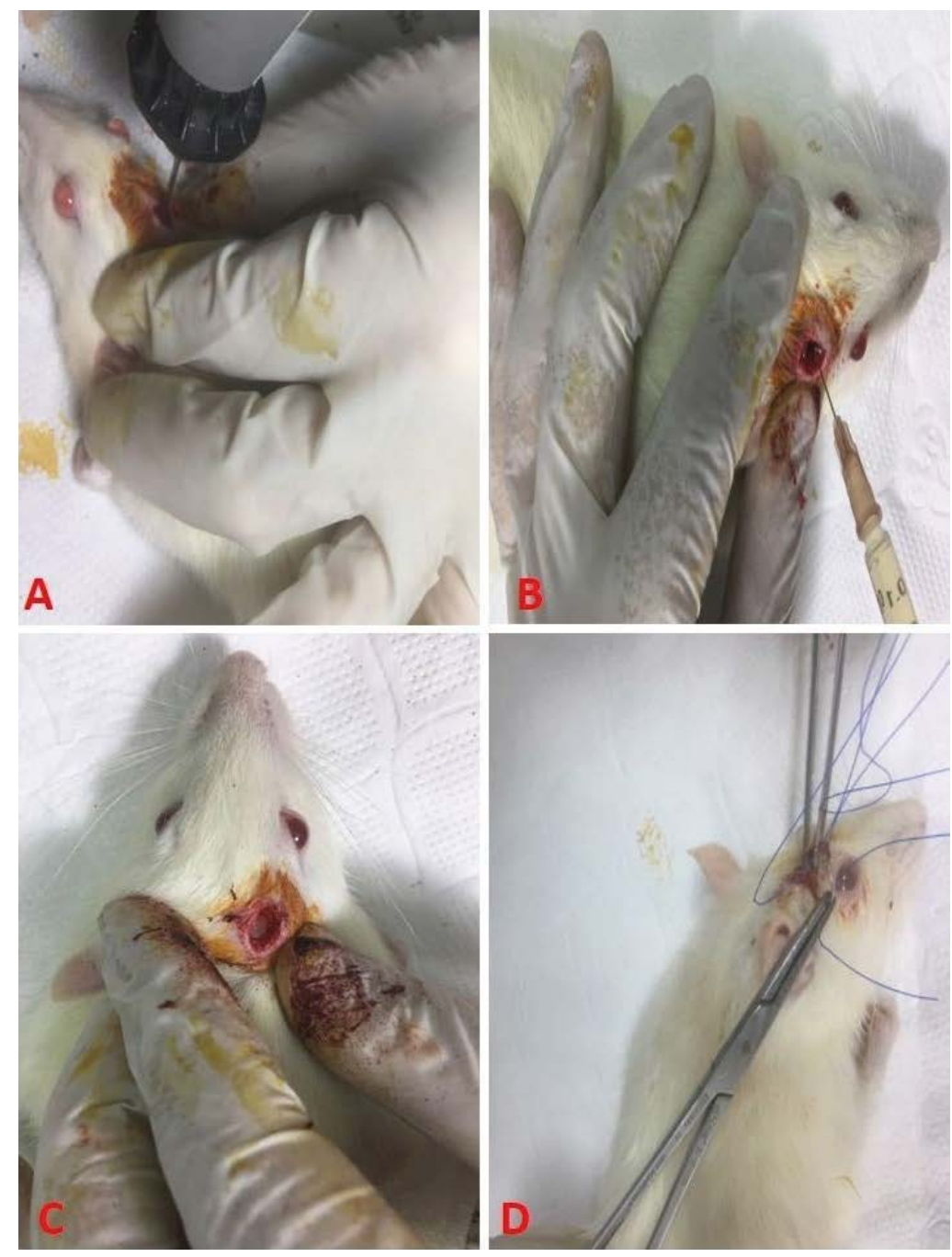

Figure 1. The process of establishing the animal model of bone defect as burr hole on the right side of the rat calvarium using a drill with $3 \mathrm{~mm}$ in diameter.

surgical procedures for a long time, comparatively through histochemical, immunohistochemical and scintigraphic studies.

\section{MATERIALS AND METHODS}

\section{Animals and experimental groups}

In this study, 30 adult $200 \mathrm{~g}$ female Wistar albino rats were used for the experiment. The experimental protocol was approved by the Ethical Committee of Aydın Adnan Menderes University (HADYEK $64583101 / 2016 / 78)$. Animals were housed in rat cages in standard conditions $\left(24 \pm 2^{\circ} \mathrm{C}\right.$ and $50 \pm 5 \%$ humidity), exposed to $12: 12$-h light/dark cycle, fed with standardised rodent chow and tap water ad libitum access. Rats were divided into three groups as intact control group ( $\mathrm{n}=10)$, bone wax group $(n=10)$, and ABS haemostat group $(n=10)$. Then, critical-sized burr-hole defects (diameter: $3.0 \mathrm{~mm}$ ) were produced in the calvarium of all rats under general anaesthesia. At the end of the 8 weeks all animals were sacrificed following scintigraphic study and cranial tissue samples were taken from the defects for histological histochemical and immunohistochemical analyses.

\section{Surgical procedure for burr hole production in rat calvarium}

In the current study, all surgical procedures were performed under general anaesthesia provided with intraperitoneal combination of ketamine $(50 \mathrm{mg} / \mathrm{kg}$ ) and xylasine ( $5 \mathrm{mg} / \mathrm{kg}$ ) anaesthesia. Following heads of rats were shaved in an area of about $2 \mathrm{~cm}$ in diameter and cleaned with baticon, a hole $3.0 \mathrm{~mm}$ in diameter was opened on the right sides of the rats' calvarium under sterile conditions, using an electric drill (CLASS Mini Grinder sets, PRC), and then the wound was closed, as described previously by Başaloğlu et al. (2021) [3] (Fig. 1). 


\section{Three-phase bone scintigraphy}

Before sacrification of the animals for histological examination, one rat was randomly selected from each group for scintigraphic study of the calvarium defect. Dynamic images were obtained immediately after the injection of $37 \mathrm{MBq}$ Tc-99m methylene diphosphonate (MDP) into the tail vein and static images were obtained after 2-h of injection with a double-headed Siemens syngo via gamma camera. Static images were acquired with a parallel hole collimator in a $256 \times 256$ matrix as 500 Kcount anterior and posterior views. Then, 10-min images were obtained from the rats' calvarial defect regions with a pin hole collimator and one of them was used as a representative figures of the groups. Tracer uptake was evaluated semiquantitatively in addition to visual evaluation. Region of interest was defined around the extracted side and the accumulation of Tc-99m-MDP in the regions was measured by software programme.

\section{Histological examination of rat calvarium}

After sacrification, the calvarial defects of the rats were taken into $10 \%$ formalin solution for $24 \mathrm{~h}$ before decalcification process using 20\% formic acid for 2 months. When softening of the tissue was acceptable tissues were processed with routine histological protocols and embedded in paraffin. Five $\mu \mathrm{m}$ sections were obtained by Leica RM 2145 microtome and after deparaffinisation haematoxylin and eosin (H\&E), Mallory's trichrome staining and anti-BSP (bs4729R, Bioss, 1:100) immunohistochemical staining were applied for evaluation of the effects of bone wax on bone osteogenesis in comparison with ABS haemostat. All slides were examined at 10-20x magnification using an Olympus C5050 camera attached to Olympus BX51 light microscope.

\section{Histomorphometry}

For histomorphometric analysis, hole diameter measurements and fibrotic scar areas in the sections were evaluated. Measurement for new ossification areas and fibrotic scar areas was also calculated using Image Pro Express 4.5 (Media Cybernetics, Inc., Rockville, MD, USA) programme. Thus, the effects of bone wax upon bone healing were compared with those of ABS haemostat.

\section{Statistical analyses}

Data analysis was performed using IBM SPSS (Statistical Package for Social Sciences) Statistics for
Windows, Version 25.0. (IBM Corp. Released 2017, Armonk, NY). Comparison between groups was made using the one-way analysis of variance method. Normality control was performed using the Shapiro-Wilk test, one of the obtained error estimates. Since there was no problem of adaptation to normal distribution, the study continued with one-way analysis of variance. Variance homogeneities were examined with Levene test, when there was no homogeneity problem, multi-group comparison was performed with F test; Bonferroni test was used as post hoc tests. In cases where variance homogeneity was not provided, Welch's test was used for multi-group comparison, Dunnett's T3 method was preferred as the post hoc test. All hypothesis checks were carried out at the 0.05 significance level, so $p<0.05$ was considered significant.

\section{RESULTS}

\section{Macroscopic examination}

After the scalp of the rats was skinned, the holes opened in the calvarium were observed macroscopically. When the skull dissection was performed macroscopically, images belonging to all groups were taken. Accordingly, it was determined that the control group was in a normal physiological recovery process. In the bone wax group, it was determined that the material filled the hole and kept the hole closed without any changes during the elapsed time. In the ABS haemostat group, it was observed that the hole opened was closed in appearance and was better than the control group in terms of vascularisation (Fig. 2).

\section{Microscopic examination}

Orientation of the hole in the bone was adjusted in microscopic sections and serial sections were taken. H\&E and Mallory's trichrome staining and anti-BSP immunohistochemical staining were performed to show the general structure in these sections. Importantly, these puncture structures were found to be filled with irregular dense connective tissue called fibrotic in both the control group and the ABS haemostat group (Fig. 2).

In the bone wax group, it was observed that there was fibrotic connective tissue in the area between the bone wax and the bone tissue at the edge of the hole where the material closed the hole, and there was no fibrotic tissue in the bone wax area. During histological follow-up, this bone wax material was seen as a gap in this area in the poured sections (Fig. 3). 

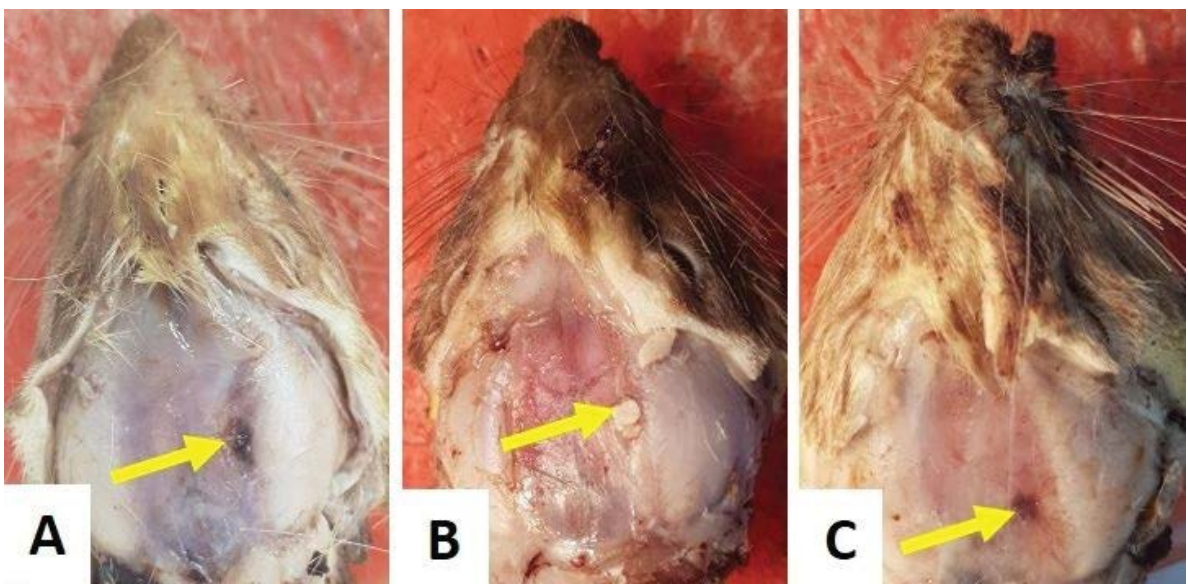

Figure 2. Demonstration of the experimentally produced bone lesions in all rat groups; A. Control group; B. Bone wax group; C. Ankaferd Blood Stopper haemostat group.
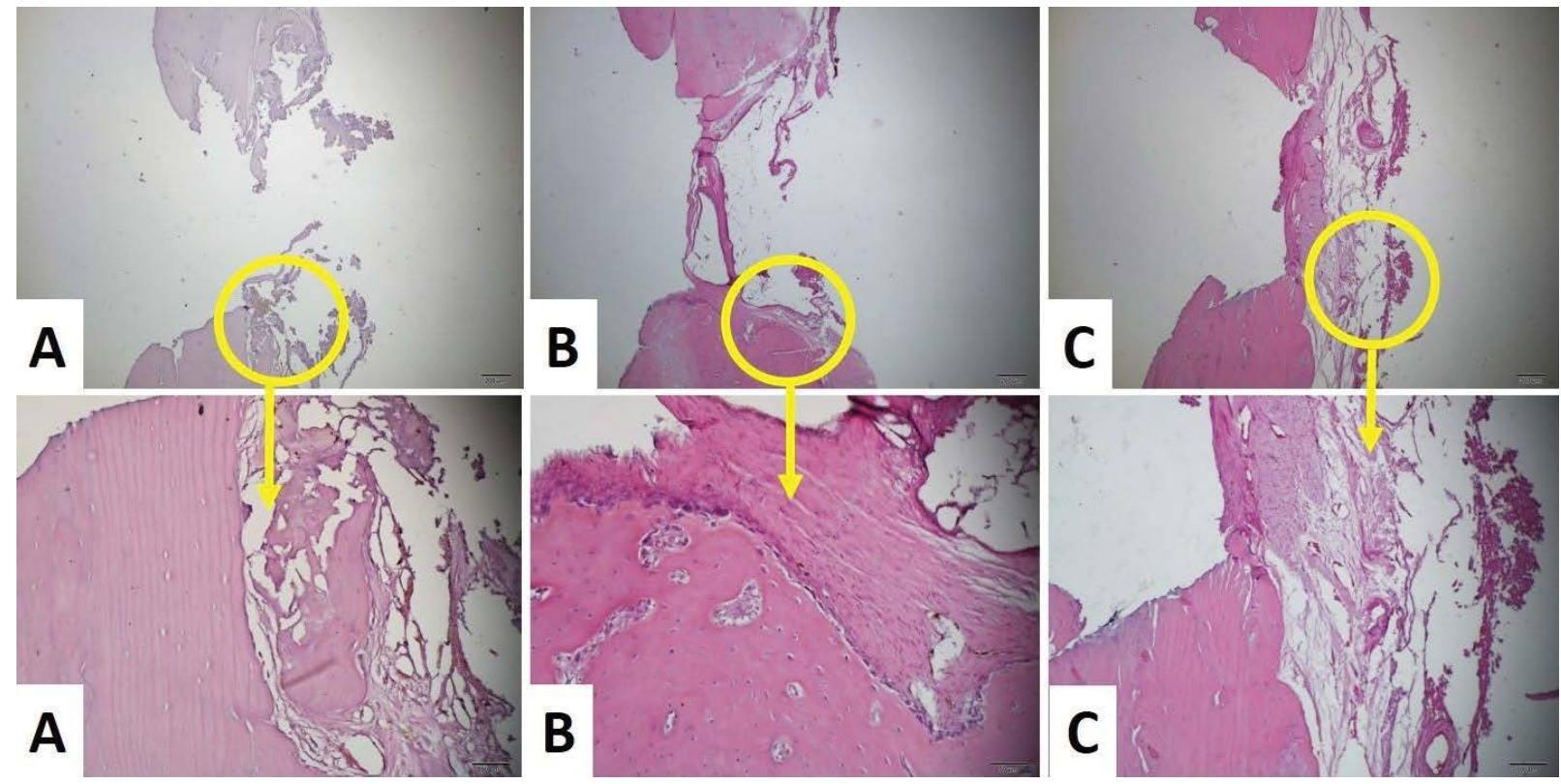

Figure 3. Microscopic evaluation of all groups; A. Control group; B. Bone wax group; C. Ankaferd Blood Stopper haemostat group. Haematoxylin and eosin staining.

When the fibrotic connective tissue structure was examined at higher magnification, it was found that they contained fibroblast-like cells and type I collagen bundles. In the control group, new bone spicules were found, probably formed by intramembranous ossification, where they settled in different regions within the connective tissue. More importantly, it was noted that this connective tissue area was small in the ABS haemostat group and ossification developed in most of the structure. With this appearance, it became the experimental group closest to the normal histological structure of the other groups. Mallory's trichrome staining showed that type I collagen bundles were stained blue, while the newly formed bone areas were red (Fig. 4). When the hole diameters were evaluated statistically, it was seen that the bone wax group had the largest hole diameter. It was found that the smallest hole diameter was in the ABS haemostat group. When the groups were compared with each other, a significant difference was found between control group and ABS haemostat group $(p<0.001)$ as well as bone wax group and $A B S$ haemostat group ( $p<0.001$ ) (Fig. 5A).

When evaluated in terms of fibrotic scar area, it was found that the lowest scar area was in the bone 


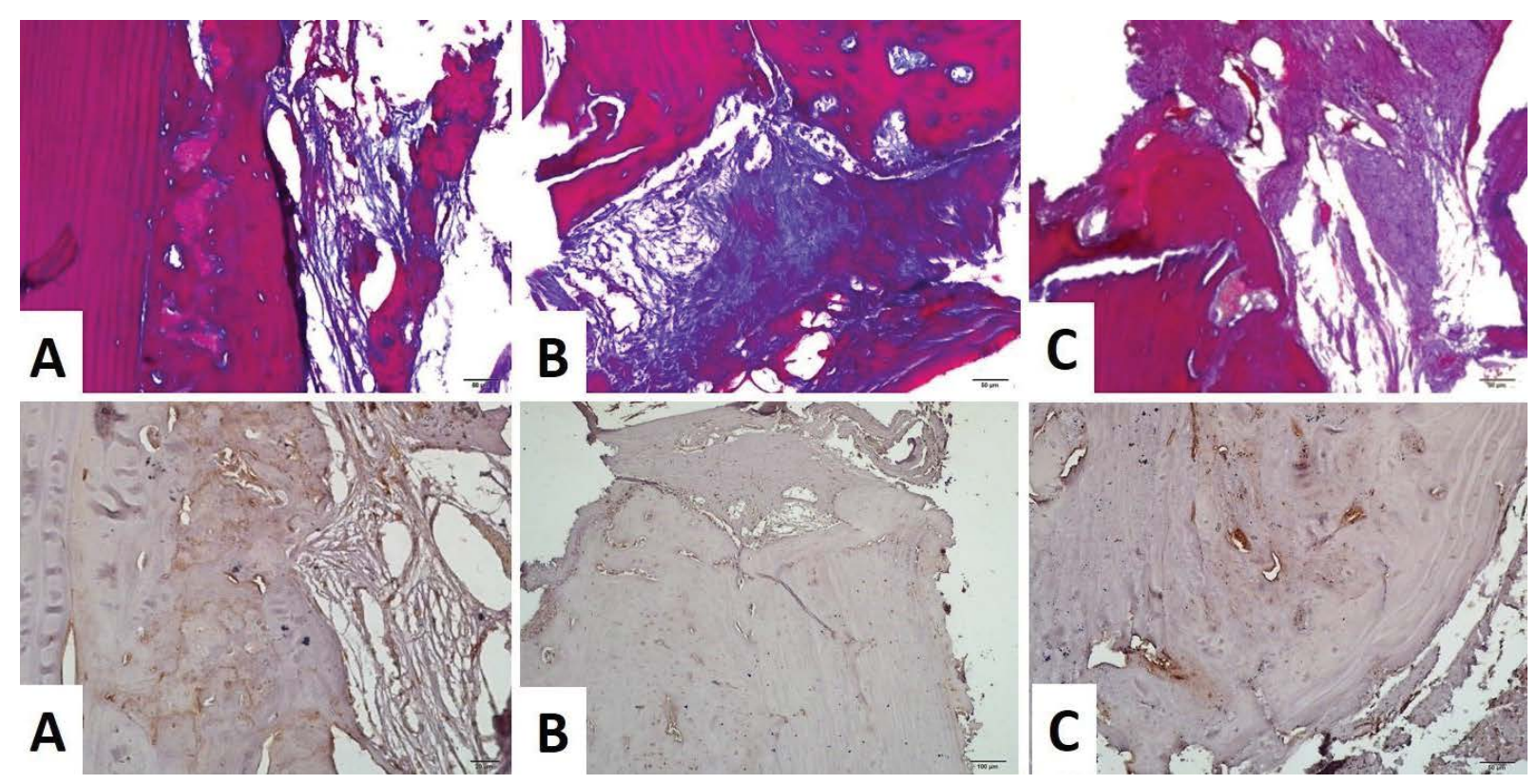

Figure 4. Microscopic evaluation of all groups showing; A. Control group; B. Bone wax group; C. Ankaferd Blood Stopper haemostat group. Top row: Mallory's trichrome staining; Bottom row: Anti-bone sialoprotein staining.
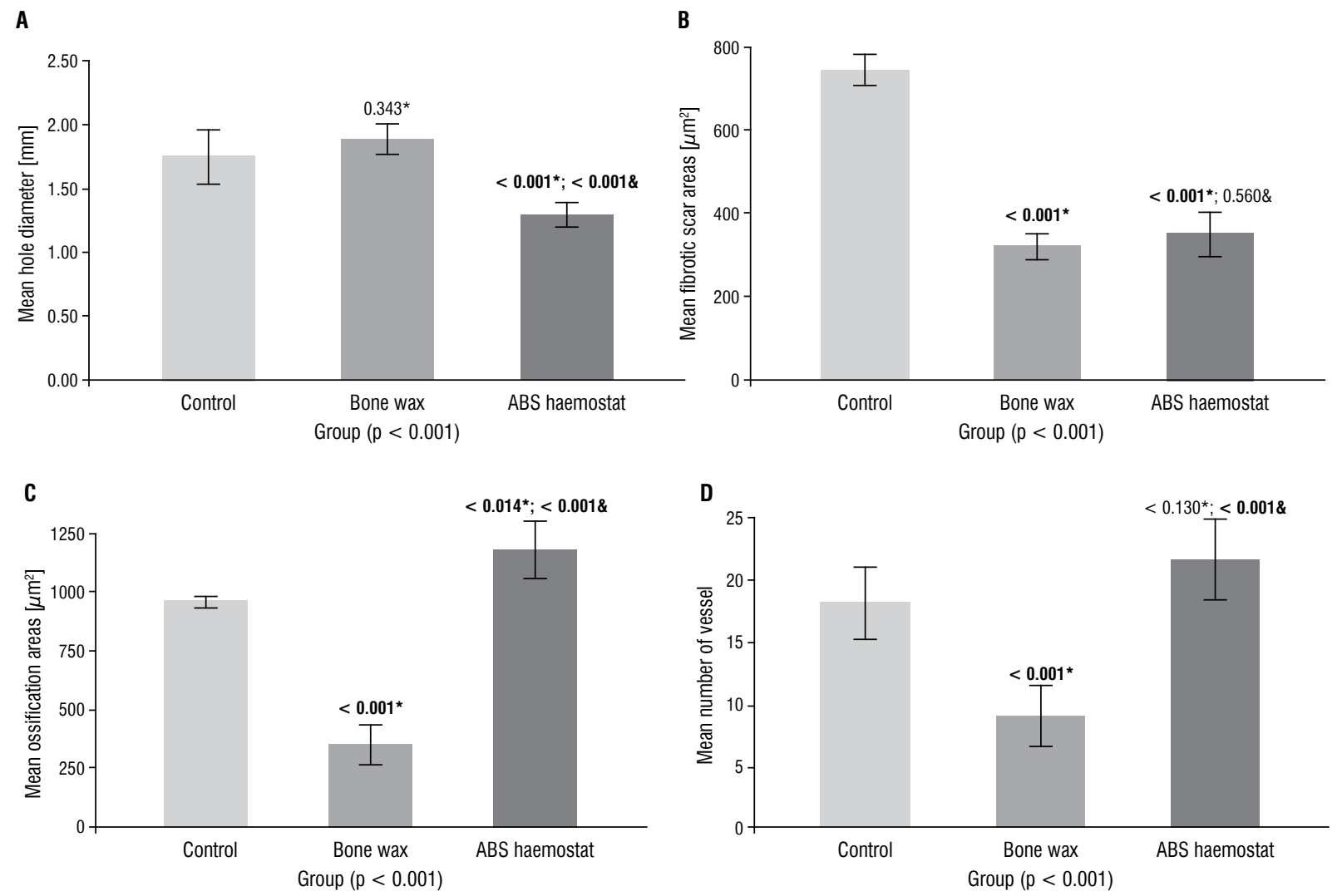

Figure 5. Statistical evaluation graph of histomorphometric analysis. Graphical representation of hole diameter (A), fibrotic scar area (B), ossification area (C) and number of vessels (D) in all groups; *Comparison with control group; \& Comparison with bone wax group.

wax group. We think that the reason for this is that the bone wax material has not been lost yet, which delays wound healing. There is a statistical signifi- cance between control group and bone wax group $(p<0.001)$ as well as bone wax group and ABS haemostat group $(p<0.001)$ (Fig. 5B). When eval- 
A

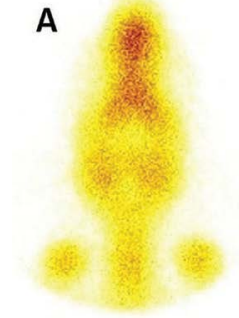

20.000 [ Left

$18.000-\quad$ Light

$16.000-$

14.000

$12.000-$

$10.000-$

$8.000-$

6.000

4.000

$2.000-$

0
B
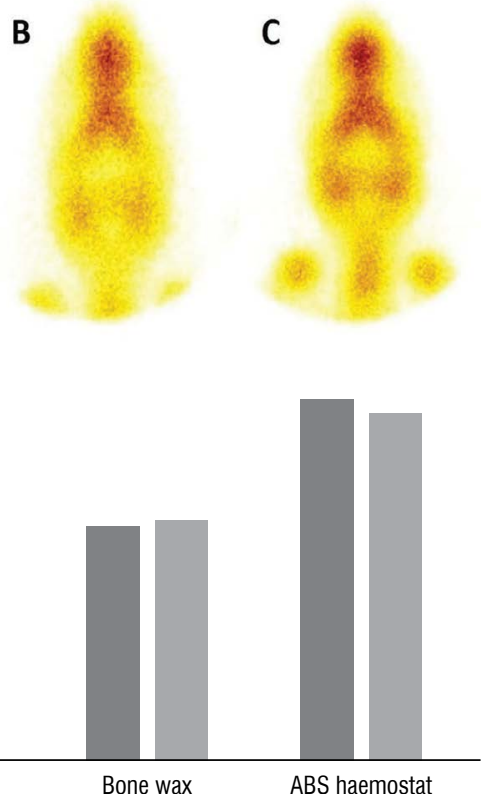

Figure 6. Scintigraphic imaging in rats; A. Control group; B. Bone wax group; C. Ankaferd Blood Stopper (ABS) haemostat group. Results of scintigraphic measurements show more pronounced osteoblastic activity depending on blood flow. In the lower part, the results obtained are also shown graphically.

uated in terms of ossification areas, it was seen that the most ossification area was in the ABS haemostat group, while the least ossified area was in the bone wax group. There is a statistical significance between control group and bone wax group $(p<0.001)$ as well as bone wax group and ABS haemostat group $(p<0.001$ ) (Fig. 5C). In terms of vascularisation, among the scar areas and ossification areas, it was seen that the most vascular structure was in the ABS haemostat group and the least in the bone wax group. The difference between control group and bone wax group $(p<0.001)$ as well as bone wax group and ABS haemostat group $(p<0.001)$ was found to be significant (Fig. 5D).

\section{Bone scintigraphy}

As a result of quantitative examination of the right and left parietal regions of the calvarium of the rats: in the rat in the intact control group, left 10.158 count, right 10.541 count; in bone wax group, left 12.008 count, right 12.325 count; and in the rat in the group with application of ABS haemostat, 18.513 count on the left was found and 17.786 count on the right (Fig. 6). In the same rat, however, no significant result was obtained in terms of vascularity richness in ossification in the right and left parietal regions. Furthermore, a significant amount of adhesion was detected in the rats in ABS haemostat group when compared to that of other two groups. The images were obtained with the pin-hole collimator in each group and they were evaluated visually for vascularity and osteoblastic activity of the region. Based on the results of scintigraphic measurements, osteoblastic activity related to blood flow in the subject taken from the group with application of ABS haemostat was more pronounced compared to that of the other two groups (Fig. 6).

\section{DISCUSSION}

The experimental design, in which the rat was used as a model, as in many other experimental studies due to its advantages in terms of ease of purchase and maintenance, followed the research line established by Başaloğlu et al. [3]. Our results clearly showed that osteoblastic activity related to blood flow was evident in the scintigraphy taken from animal with application of ABS haemostat and histological findings confirmed the positive effect of the ABS haemostat application upon the bone healing, while bone wax as a haemostatic agent had a negative impact on the bone regeneration. Also, a statistical difference was found between the groups for the area of fibrotic connective tissue $(p<0.05)$.

The cranium, mostly composed of symmetrical and paired bones, is the most important bone area of the body because it is the main defence system of the brain and brain stem [28]. We can say that it is biologically more inert because it has a different blood supply structure than bone marrow and other bones [22]. It protects the brain from the central nervous system elements that ensure regular and harmonious working coordination of vital functions. Therefore, there are many publications and experimental studies about the healing of cranial defects in the current literature.

When the literature was reviewed on how these substances used in the control of bleeding affect bone healing, it has been determined that significant differences can be detected in first 30 days $[1,9,15]$. In animal experiments, critical dimensional defect was defined as the smallest size bone wound that did not heal spontaneously throughout life without using any material $[8,25]$. Such defects tend to fill with fibrous connective tissue and heal this way rather than by bone filling $[10,24]$. In their study, Turnbull and 
Freeman (1974) [30] examined the 2-mm-diameter experimental defects in the parietal bones of rats in terms of healing for 12 weeks and it was observed that there was no significant improvement at the end of this period. Mulliken and Glowacki (1980) [19] examined $2 \mathrm{~mm}$ parietal bone defects. While they could not find a bone filling at the end of the $6^{\text {th }}$ month in control defects, they reported that they encountered bone formation in the experimental group where they applied demineralised bone powder [19].

Howard and Kelly (1969) [15] found that bone wax negatively affected healing in an experimental study they performed in rat tibia. They found that while there was almost complete recovery in the control group of rats that were sacrificed on day 31 , the material was not resorbed and delayed osteoblastic activity in the group treated with bone wax [25]. Nooh et al. (2014) [21] used two haemostatic agents, surgicel and bone wax, in the bone defects formed in the lateral direction of the right and left metacarpal of goats. Sacrificed animals were subjected to computed tomography and histological examinations at 3 and 5 weeks. As a result, they stated that the surgicel group was superior to the bone wax group in terms of bone healing, and bone wax prevented osteogenesis and caused inflammation [21]. Raposo-Amaral et al. [23] performed histological and radiological examination of the use of bone wax in rats with cranial defects. After the surgery, computed tomography scan of the rats was performed at $12^{\text {th }}$ week and as a result, they found that bone wax significantly reduced the bone rate, caused $18 \%$ in the healing of the cranial defect and caused fibrosis with infection in rats [23]. Similar results were obtained in animals treated with bone wax in our study, although the drill-opened defect area appeared to be closed macroscopically, this area melted after acid treatment and little fibrotic tissue remained. This situation suggests that bone wax prevents mineralisation. In this group, the intensity of osteoclastic activity along with osteoblastic activity is also noteworthy. Bone regeneration and bone resorption continue almost simultaneously. In intact control group, very few new bone spicules were encountered due to intramembranous ossification. The fact that intact control group has a higher ossification pattern compared to bone wax group suggests that it is significant in terms of revealing the negative effect of bone wax on ossification again. This situation is again consistent with similar studies in the literature [11].
On the other hand, however, there are quite a few studies in the literature regarding bone healing, both early and late, and similar to our study, there are also literature studies involving advanced bone healing. In previous histological studies investigating the effects of $A B S$ on the bone tissue, it was found to have an acceleratory effect on the short-term bone healing process in samples taken on the $7^{\text {th }}$ postoperative day [16, 27]. Recently, Kuruoğlu et al. (2017) [17] evaluated the late effects of the combination of ABS and $\beta$-tricalcium phosphate (TCP) using immunohistochemical methods and they found the bone healing effects of $A B S$ haemostat in their study. Afterwards, Tanık et al. (2018) [29] also investigated the late period effects of the combination of ABS and $\beta$-TCP on the regeneration of bone tissue in rats. They found that both $\beta-T C P$ and ABS had positive histological and radiological effects on wound healing and bone formation in samples taken on day 56 after surgery [29]. In the first macroscopic examination we observed in our study, it was determined that the group with the appearance of scar tissue most closely resembling normal structure was the group with application of $A B S$ haemostat, suggesting a positive effect of $A B S$ haemostat on wound healing.

Histologically, in the Mallory's trichrome staining, type I collagen bundles were stained in blue, and the newly formed bone areas were red in all groups in our study. In terms of hole diameters, it was determined that the bone wax group had the largest hole diameter and the ABS haemostat group had the smallest hole diameter. A statistically significant difference was found between control group and ABS haemostat group $(p<0.001)$ as well as bone wax group and ABS haemostat group $(p<0.001)$. When evaluated in terms of fibrotic scar area, it was found that the lowest scar area was in the bone wax group. We think that the reason for this is that the bone wax material has not been lost yet, which delays wound healing. There is a statistical significance between control group and bone wax group $(p<0.001)$ as well as bone wax group and ABS haemostat group $(p<0.001)$. In the literature review, however, immunohistochemically studies on bone repair of ABS haemostat are limited. In their experimental study in which they evaluated the use of platelet-rich plasma together with autogenous bone graft in the repair of bone defects by immunohistochemical analysis, Nagata et al. (2009) [20] found that the expression of OC and $\mathrm{OP}$ was significantly higher in the platelet-rich plasma 
group. In our study, ossification is present in all three groups, although it seems that the mineralisation has not yet been completed. Therefore, absence of any analysis for inorganic material content of bone tissue mineralisation and difference of drilled defects from those in humans in terms of bone remodelling are limitations of our study. Moreover, lack of any imaging or mechanical study investigating the bone healing is another limitation of the study. Future studies including immunohistochemical determination of bone related proteins in the early stages of bone healing are needed for better understanding of impact of the ABS haemostat application upon bone remodelling apart from the quick stop of haemorrhage.

It is well-known that three-phase bone scintigraphy is a non-invasive technique showing the vascularity and osteoblastic activity of the bone. Based on their findings in a study, Aygit et al. (1999) [2] stated that bone scintigraphy could be used as a noninvasive method in evaluating implant vascularity because histological changes and scintigraphic findings were parallel. Tc-99m MDP is a radiopharmaceutical that can chemically adhere to all bones in the skeletal system after intravenous administration. It shows a significant accumulation especially at the points where bone metabolism increases. The common features of radiopharmaceuticals such as MDP, hydroxydiphosphonate (HDP) and hydroxymethylene diphosphonate (HMDP) marked with Tc-99m are that the diphosphonate core in their structure acts like hydroxyapatite, which is the basic building block of bone, and accumulates reversibly on bone surfaces and shows osteoblastic activity depending on blood flow. In this way, all bones belonging to the skeletal system get rid of the soft tissue effect and become visible in a plain state [26]. Diphosphonates attach to hydroxyapatite crystals, one of the main components of the bone matrix, by physicochemical absorption. This amount of adhesion varies according to the blood supply and osteoblastic activity in that area. Organization of haemostasis is of primary importance in fracture or defect repair. When scintigraphic imaging was performed there was no clear difference between the groups. Although MDP is a chemoabsorbable substance in all bones of the skeletal system, it shows a significant accumulation in places where bone metabolism is increased. Radiopharmaceuticals such as MDP, HDP, and HMDP act as hydroxyapatite, which is the main component of bone, and show osteoblastic activity due to a reversible blood flow at the bone surfaces [28]. Although there are not many such studies in the literature, we think that performing bone scintigraphy in weekly periods starting from the defect opening will be more beneficial as it will provide comparable and observable data.

\section{CONCLUSIONS}

Bone fractures due to trauma or surgical operations are conditions that can be encountered in all stages of life. The primary functions of ABS haemostat and bone wax, which are used as haemostatic agents in neurosurgery, orthopaedic and traumatology surgery, oral and maxillofacial surgery, are to stop bleeding quickly. Taken as whole, our results showed that: (1) Histological, immunohistochemical and bone scintigraphic data support the positive effects of $A B S$, consisting of folkloric medicinal plant extracts, on bone healing as well as stopping bleeding rapidly. It was concluded that ABS haemostat also had a positive effect on bone regeneration, while bone wax had a negative effect on regeneration of the rats' calvarial defects; (2) We think it is important in various surgical procedures, to choose a blood stopping agent, such as ABS, that will both provide rapid control of bleeding and accelerate regeneration by helping bone repair. However, the effectiveness of this product as a therapeutic modality for possible future clinical applications should be evaluated for all types of bones, including extracranial, spongy and cortical, apart from cranial ones; (3) Furthermore, this study also suggests that the anti-inflammatory and antimicrobial properties of the plant ingredients of antihaemorrhagic ABS should be evaluated in terms of preventing various complications during the operation and postoperative period in future studies.

\section{Acknowledgements}

The authors are grateful to Mr. Rifat Aydın for technical support and Aydın Adnan Menderes University Scientific Research Projects (BAP) Unit for financial support. We would like to thank Prof. Ali Akhaddar for his suggestions following review of the article draft.

\section{Conflict of interest: None declared}

\section{REFERENCES}

1. Alberius $P$, Klinge $B$, Sjögren $S$. Effects of bone wax on rabbit cranial bone lesions. J Craniomaxillofac Surg. 1987; 
15(2): 63-67, doi: 10.1016/s1010-5182(87)80020-3, indexed in Pubmed: 3294903.

2. Aygit AC, Sarikaya A, Candan L, et al. Comparison of alloplastic implants for facial bones by scintigraphy and histology: an experimental study. Eur J Plast Surg. 1999; 22(2-3): 102-106, doi: 10.1007/s002380050158.

3. Basaloglu HK, Turgut $M$, Şirin $C$, et al. The influence of functional pinealectomy and exogenous melatonin application on healing of burr hole in adult rat calvaria: a histological and immunohistochemical study. Folia Morphol. 2022 [Epub ahead of print]; 81(2): 271-279, doi: 10.5603/FM.a2021.0047, indexed in Pubmed: 33997947.

4. Bulut E, Baş B, Altunkaynak BZ, et al. Efficacy of Ankaferd Blood Stopper on bone healing in diabetic rats: a stereological and histopathological study. Biotech Histochem. 2014; 89(7): 535-543, doi: 10.3109/10520295.2014.906657, indexed in Pubmed: 24799094.

5. Ciftciler R, Ciftciler AE, Malkan UY, et al. Pharmacobiological management of hemostasis within clinical backgrounds via Ankaferd hemostat (Ankaferd blood stopper). SAGE Open Med. 2020; 8: 2050312120907811, doi: 10.1177/2050312120907811, indexed in Pubmed: 32110403.

6. Çiftçiler R, Haznedaroglu iC. Ankaferd hemostat: from molecules to medicine. Turk J Med Sci. 2020; 50(SI-2): 1739-1750, doi: 10.3906/sag-1908-161, indexed in Pubmed: 32283900.

7. Cipil HS, Kosar A, Kaya A, et al. In vivo hemostatic effect of the medicinal plant extract Ankaferd Blood Stopper in rats pretreated with warfarin. Clin Appl Thromb Hemost. 2009; 15(3): 270-276, doi: 10.1177/1076029608329578, indexed in Pubmed: 19117967.

8. Cook JL, Hung CT, Kuroki K, et al. Animal models of cartilage repair. Bone Joint Res. 2014; 3(4): 89-94, doi: 10.1302/20463758.34.2000238, indexed in Pubmed: 24695750.

9. Finn MD, Schow SR, Schneiderman ED. Osseous regeneration in the presence of four common hemostatic agents. J Oral Maxillofac Surg. 1992; 50(6): 608-612, doi: 10.1016/02782391(92)90443-4, indexed in Pubmed: 1593323.

10. Frame JW. A convenient animal model for testing bone substitute materials. J Oral Surg. 1980; 38(3): 176-180, indexed in Pubmed: 6928181.

11. Geary JR, Frantz VK. New absorbable hemastatik bonewax; experimental and clinical studies. Ann Surg. 1950; 132(6): 1128-1137, doi: 10.1097/00000658-195012000-00012, indexed in Pubmed: 14790584.

12. Goker H, Haznedaroglu IC, Ercetin S, et al. Haemostatic actions of the folkloric medicinal plant extract Ankaferd Blood Stopper. J Int Med Res. 2008; 36(1): 163-170, doi: 10.1177/147323000803600121, indexed in Pubmed: 18304416.

13. Gül M, Günay A, Tanik A. An evaluation of the effects of caffeic acid phenethyl ester and Ankaferd blood stopper on secondary wound healing of oral mucosal tissue. Turk J Med Sci. 2020; 50(1): 248-257, doi: 10.3906/sag-1908114, indexed in Pubmed: 31769639.

14. Gupta G, Prestigiacomo CJ. From sealing wax to bone wax: predecessors to Horsley's development. Neurosurg Focus. 2007; 23(1): E16, doi: 10.3171/foc.2007.23.1.16, indexed in Pubmed: 17961057.

15. Howard TC, Kelley RR. The effect of bone wax on the healing of experimental rat tibial lesions. Clin Orthop Relat Res. 1969; 63: 226-232, indexed in Pubmed: 5781118.

16. Işler SC, Demircan S, Cakarer S, et al. Effects of folk medicinal plant extract Ankaferd Blood Stopper on early bone healing. J Appl Oral Sci. 2010; 18(4): 409-414, doi: 10.1590/s167877572010000400015, indexed in Pubmed: 20835578.
17. Kuruoglu E, Onger ME, Marangoz AH, et al. Postlaminectomy bone and scar formations in presence of Ankaferd Blood Stopper and Bitter Melon (Momordica Charantia): An experimental study. Turk Neurosurg. 2017; 27(3): 441-446, doi: 10.5137/1019-5149.JTN.16263-15.1, indexed in Pubmed: 27593811.

18. Malaval L, Wade-Guéye NM, Boudiffa M, et al. Bone sialoprotein plays a functional role in bone formation and osteoclastogenesis. J Exp Med. 2008; 205(5): 1145-1153, doi: 10.1084/jem.20071294, indexed in Pubmed: 18458111.

19. Mulliken JB, Glowacki J. Induced osteogenesis for repair and construction in the craniofacial region. Plast Reconstr Surg. 1980; 65(5): 553-560, doi: 10.1097/00006534198005000-00001, indexed in Pubmed: 6988853.

20. Nagata M, Messora M, Okamoto R, et al. Influence of the proportion of particulate autogenous bone graft/platelet-rich plasma on bone healing in critical-size defects: an immunohistochemical analysis in rat calvaria. Bone. 2009; 45(2): 339-345, doi: 10.1016/j.bone.2009.04.246, indexed in Pubmed: 19410024.

21. Nooh N, Abdullah WA, Grawish MEA, et al. The effects of surgicel and bone wax hemostatic agents on bone healing: An experimental study. Indian J Orthop. 2014; 48(3): 319-325, doi: 10.4103/0019-5413.129451, indexed in Pubmed: 24932041.

22. Prolo DJ, Gutierrez RV, DeVine JS, et al. Clinical utility of allogeneic skull discs in human craniotomy. Neurosurgery. 1984 ; $14(2)$ : 183-186, doi: 10.1227/00006123198402000-00011, indexed in Pubmed: 6709140.

23. Raposo-Amaral CE, Almeida AB, Paschoal G, et al. Histological and radiological changes in cranial bone in the presence of bone wax. Acta Cir Bras. 2011; 26(4): 274-278, doi: 10.1590/ s0102-86502011000400005, indexed in Pubmed: 21808839.

24. Rowe NL. Nonunion of the mandible and maxilla. J Oral Surg. 1969; 27(7): 520-529, indexed in Pubmed: 4893250.

25. Schmitz JP, Hollinger JO. The critical size defect as an experimental model for craniomandibulofacial nonunions. Clin Orthop Relat Res. 1986(205): 299-308, indexed in Pubmed: 3084153.

26. Sengöz T, Karaçalıoğlu AÖ, İnce $S$, et al. Cardiac Tc- $99 \mathrm{~m}$ metylene diphosphanate uptake in bone scintigraphy (in Turkish). Gülhane Tıp Dergisi. 2015; 57: 81-83.

27. Simşek HO, Tüzüm MŞ, Baykul T, et al. Experimental investigation of the effects of a blood stopper agent (ankaferd blood stopper) on bone surfaces. Turk J Haematol. 2013; 30(2): 177-183, doi: 10.4274/Tjh.2012.0092, indexed in Pubmed: 24385782.

28. Sirola K. Regeneration of defects in the calvaria. An experimental study. Ann Med Exp Biol Fenn. 1960; 38(Suppl 2): 1-87, indexed in Pubmed: 14447201.

29. Tanık A, Güler Doğru A, Akpolat V, et al. Investigation of the effect of combined use of alloplastic-based tricalcium phosphate bone graft and antihemorrhagic plant extract (ABS) on bone regeneration in surgically induced bone defects in nondiabetic rats: an experimental animal study. Turk J Med Sci. 2018; 48(6): 1302-1314, doi: 10.3906/ sag-1803-201, indexed in Pubmed: 30543085.

30. Turnbull RS, Freeman E. Use of wounds in the parietal bone of the rat for evaluating bone marrow for grafting into periodontal defects. J Periodontal Res. 1974; 9(1): 39-43, doi: 10.1111/j.1600-0765.1974.tb00651.x, indexed in Pubmed: 4277754.

31. Zhou H, Ge J, Bai Y, et al. Translation of bone wax and its substitutes: History, clinical status and future directions. J Orthop Translat. 2019; 17: 64-72, doi: 10.1016/j. jot.2019.03.005, indexed in Pubmed: 31194062. 